\title{
ASOCIACIONES DE MIGRANTES ANDINOS EN LA WEB. RELEVANCIA DE REDES VIRTU- ALES EN EL ESTABLECIMIENTO DE LAZOS COMUNITARIOS
}

\author{
CECILIA MELELLA \\ IMHICIHU CONICET \\ BUENOS AIRES, ARGENTINA \\ E-MAIL: CEMELELLA@GMAIL.COM
}

HTTP://DX.DOI.ORG/10.5902/2316882X17632 
Resumo: A revolução na TIC do final do século XX trouxe profundas mudanças que afetaram diversas práticas sociais, incluindo a migração. Na Argentina, a presença de associações de migrantes países andinos na Web tem levado a uma maior visibilidade e contribuiu para o funcionamento das redes sociais dos migrantes. O objetivo deste trabalho é o estudo dos sites destas associações em dois casos diferenciais. Equatorianos, que representam uma nova migração para o país e peruanos são uma mais estável.

Palavras-chave: Internet, migração, países andinos, laços comunitários, associações

\section{ASOCIACIONES DE MIGRANTES ANDINOS EN LA WEB. RELEVAN- CIA DE REDES VIRTUALES EN EL ESTABLECIMIENTO DE LAZOS COMUNITARIOS}

Resumen: La revolución de las TIC de finales del siglo XX produjo transformaciones profundas que afectaron diversas prácticas sociales, entre ellas, las migraciones. En la Argentina, la presencia de las asociaciones de migrantes de países andinos en la Web ha provocado mayor visibilidad y ha contribuido al funcionamiento de las redes sociales de migrantes. El objetivo de este trabajo es el estudio de los sitios Web de estas asociaciones sobre dos casos diferenciales. Los ecuatorianos quienes representan una migración joven hacia el país y los peruanos que constituyen una más asentada.

Palabras clave: Internet, migraciones, países andinos, lazos comunitarios, asociaciones

ANDEAN MIGRANT ASSOCIATIONS ON WEB. THE RELEVANCE OF VIRTUAL NETWORKS IN ESTABLISHING COMMUNITY TIES

Abstract: The revolution in ITC of the late twentieth century promoted deep changes in several social practices like migrations. The Web presence of Andean migrant associations in Argentina has contributed to the social networks of these groups. Thus, the objective of this work is the study of the websites of the associations of migrants from Andean countries in Argentina on two different cases. Ecuadorians who represent a younger migration to the country and Peruvians which are a more settled.

Keywords:Internet, migration, Andean countries, community ties, associations

Rev. Cad.Comun, Santa Maria, v.20,n.1, art 1, p.1 de 24, jan/abr.2016 


\section{INTRODUCCIÓN: REDES SOCIALES Y COMUNIDADES VIRTUALES}

Los avances de las Tecnologías de a información y comunicación (TIC) y de los transportes junto con la organización internacional del trabajo y del consumo y sus consecuentes movimientos poblacionales (CASTELLS, 1995) generaron cambios cualitativos respecto de la densidad, magnitud y movilidad de los vínculos y redes establecidas por los migrantes internacionales (PEÑARANDA CÓLERA, 2008). El desarraigo y la ruptura de lazos que caracterizaban las prácticas migratorias de los siglos XIX y XX han dado lugar a otras experiencias representadas por la continuidad $y$ el sostenimiento de vínculos. Se ha pasado del paradigma del migrante desarraigado al migrante conectado (DIMINESCU, 2011) representado por una doble presencia -la física (o real) y la imaginada (o virtual)- que posibilita estar aquí y allá participando de la comunidad de origen y de destino (tornando estas diferenciaciones cada vez más caducas).

Las TIC tradicionales como el correo postal, el telégrafo o el teléfono han facilitado históricamente el contacto a distancia entre las personas. Las nuevas TIC, como productos de un proceso complejo y en constante cambio, se han diversificado en distintas formas como Internet y, en particular, los e-mails, el chat, Skype y los teléfonos celulares. Estas herramientas y/o artefactos culturales (HINE, 2004) potencian y cambian los usos y las mediaciones que las personas les daban a los artefactos pasados (WINOCUR, 2007 y 2010). El teléfono se establece como el primer artefacto tecnológico utilizado por los migrantes para conectarse con sus familias (GONZÁLVEZ TORRALBO, 2012). El segundo es Internet. Desde la World Wide Web pueden contactarse con su núcleo familiar y connacionales, buscar información, participar y crear Webs en cualquier parte del mundo. El acceso ilimitado no implica desconocer la brecha digital y el nivel de conectividad. Según CEPAL (2013), en América Latina el 40\% de la población es usuaria de Internet. Mientras que en los países miembros de la Organización para la Cooperación y el Desarrollo Económico (OCDE) el acceso es del 80\% (BARRANTES, JORDÁN y ROJAS, 2013).

De esta manera, Internet resulta una tecnología capital para estos grupos al expandir de forma grandilocuente las posibilidades para la conformación de redes transnacionales sobre la base de comunidades virtuales (GÓMEZ ESCALONILLA Y CAMPOS ZABALA, 2009). Pertenecer a una red significa trabajar con otros formando parte de un proceso de flujos de in-

Rev. Cad.Comun, Santa Maria, v.20,n.1, art 1, p.1 de 24, jan/abr.2016 
formación, comunicación e intercambio de experiencias que altera la forma piramidal jerárquica (QUINTAR, 2007). El ciberespacio acarrea la renovación de la idea de comunidad virtual como punto de pasaje de creencias y prácticas compartidas que vinculan a las personas físicamente separadas (PISCITELLI, 2002). Las ciber-comunidades evidencian un cambio en el mismo concepto de comunidad que pasa del lugar físico a la red virtual. Sin embargo, como sostiene Piscitelli "la tecnología no remedia los males de la biología ni de la cultura, sino que las complementa y las amplifica -para mejorarlas o para empeorarlas-" (PISCITELLI, 2002: 83). En consecuencia, por un lado, las comunidades electrónicas son comunidades liberadas (FINQUELIEVICH, 1999) al no estar encadenadas a los límites espacio-temporales. Por otro lado, éstas no dejan de relacionarse con lo local más que con lo global y fortifican las redes sociales existentes, como es el caso de algunas asociaciones de migrantes en la Argentina.

En la Argentina, el uso y apropiación de la Internet por parte de los colectivos de migrantes ha permitido el desarrollo de diferentes prácticas como la prensa de migrantes on line, los sitios Web de asociaciones políticas, culturales y sociales y la presencia heterogénea en la red Facebook. Particularmente, la presencia de las asociaciones de migrantes en la Web ha contribuido a la conformación y funcionamiento de las redes sociales, por lo general, sin la intervención y/o mediación del Estado y ha brindado soporte a las transformaciones políticas, familiares, comunitarias, culturales y económicas en los grupos migratorios transnacionales (PORTES, 2005).

Por consiguiente, el estudio de los sitios Web de las asociaciones de migrantes de países andinos en la Argentina permite, por un lado, analizar sus potencialidades y alcances. Por otra parte, nos autoriza a reflexionar sobre la tecnosociabilidad (los cambios culturales vinculados a las TIC) y la creación de lazos en una era donde la definición del migrante pareciera dejar atrás la territorialidad en pos de la conformación de diásporas virtuales (DIMINESCU, 2011). Seleccionamos las colectividades de países andinos pues conforman una migración que se ha proyectado hacia la Argentina fuertemente desde la década de 1990. Además, éstas exponen dos tipos de migración, una reciente y joven, representada por los colombianos y ecuatorianos y otra tradicional y afincada, representada por los bolivianos y peruanos. Ambos grupos generan prácticas sociales, políticas y culturales diferentes en las sociedades receptoras como las festividades cívicas

Rev. Cad.Comun, Santa Maria, v.20,n.1, art 1, p.1 de 24, jan/abr.2016 
o religiosas, la fundación de asociaciones, la distribución de periódicos, entre otras. Consecuentemente, proponemos que el uso y la apropiación de Internet también presentan sus diferencias según cada colectivo.

\section{METOdOLOGÍA ¿CÓMO ANALIZAR LAS REDES SOCIALES VIRTU- ALES? UNA PERSPECTIVA INTERDISCIPLINARIA}

El objetivo principal de este trabajo es, por un lado, analizar la presencia de las asociaciones de migrantes de países andinos de la Argentina en la Web y, por otro lado, estudiar sus posibilidades de conectividad y de formación de redes sociales. Ahora bien, la misma definición de Internet como espacio y como herramienta nos presenta el desafío metodológico de su abordaje donde su caracterización como objeto de estudio e instrumento de investigación se solapan constantemente (ESTALELLA Y ARDÉVOL, 2010). Entendemos Internet desde una doble dimensión. Por un lado, como artefacto cultural, una tecnología, una herramienta que adquiere significados culturales y usos diferentes en relación a los contextos donde es utilizada. Por otro lado, como un lugar, un espacio donde se gesta una cultura (HINE, 2004).

Dada esta doble condición, utilizamos una metodología fundamentalmente cualitativa que combine diversos saberes desde una perspectiva interdisciplinaria. Para analizar los sitios Web como un lugares o espacios donde se gesta una cultura (HINE, 2004) consideramos, en primer lugar, a los portales como textos. Éstos son el resultado de discursos sociales que fueron cristalizados por el analista para su estudio (VERÓN, 1993). La teoría de los discursos sociales elaborada por el semiólogo argentino Eliseo Verón (1993) concibe a los fenómenos de sentido como conglomerados de materias significantes remitentes a una red semiótica conceptualizada como sistema productivo. Se parte de la conformación de una teoría general de la producción de sentido donde se articulan las dimensiones de la producción, de la circulación y del consumo o reconocimiento. El discurso es un punto de pasaje, un encuentro entre el conjunto de las relaciones de producción y de reconocimiento de los fenómenos de sentido. Los discursos (cristalizados como textos) están constituidos por un conjunto de marcas y/o rasgos de tres tipos: retóricos, temáticos y enunciativos.

Así, desde un enfoque semiótico recurrimos al análisis de discurso y contenido de los sitios Web de asociaciones considerados como textos y

Rev. Cad.Comun, Santa Maria, v.20,n.1, art 1, p.1 de 24, jan/abr.2016 
compuestos por tres dimensiones (retórica o figural, temática y enunciativa) que reflejan su modo de hacer, su estilo. Así, detectamos elementos textuales y paratextuales que nos permitan dar cuenta de las estrategias retóricas, temáticas y enunciativas principales de cada uno de los sitios seleccionados. La superficie retórica está compuesta por los elementos esenciales que definen la estructura del texto y permiten diferenciarlo de otros (STEIMBERG, 1993; BARTHES, 1986). Características como estrategias gráficas y uso del color, presencia de fotografías, publicidades, uso y diagramación del espacio, logotipo son las estrategias discursivas que a nivel retórico configuran los sitios de los grupos de migrantes andinos. El estudio de la dimensión temática comprende la expresión de diversos temas presentes en el texto: discriminación y xenofobia, religiosidad, indigenismo, ampliación de derechos (políticos, civiles y sociales) y las políticas migratorias. El análisis enunciativo se presentó posteriormente al retórico y temático pues ambas dimensiones inciden en la conformación de la enunciación, a la cual sintetizamos como el efecto de sentido que construye una situación comunicacional. Para facilitar el análisis recurrimos al concepto de contrato de lectura de Eliseo Verón (1985) definido como la relación entre un soporte y su lectura, un nexo entre lo que se dice y las modalidades del decir.

En segundo lugar, para abordar Internet como artefacto cultural, es decir, como tecnología con propiedades específicas en un contexto de uso y apropiación (HINE, 2004) nos centramos en una perspectiva comunicacional que nos permitió examinar la estructura de cada sitio de acuerdo con la presencia de recursos tecnológicos y a las posibilidades comunicativas que éstos proponen. Tuvimos en cuenta la categorización propuesta por el catedrático argentino Luis Albornoz para su investigación sobre periódicos digitales titulada "Periodismo digital" de 2007 y consideramos la presencia en cada página Web de: contenidos informativos que incluye contenidos de la actualidad informativa, contenidos prácticos para la vida cotidiana, contenidos de entretenimiento y contenidos del propio diario servicios al lector usuario que Servicios que brinda el periódico a través de dispositivos y herramientas tecnológicas como son los buscadores, la presencia de archivos en formatos HTML, PDF o las versiones para imprimir, el envío por e-mail, entre otros. También áreas de participación y recursos audiovisuales, multimedia e hipervínculos.

Por último, para analizar la conectividad entre los diferentes portales

Rev. Cad.Comun, Santa Maria, v.20,n.1, art 1, p.1 de 24, jan/abr.2016 
recurrimos al concepto de lazo social desarrollado por la teoría de las redes, particularmente, los trabajos de Mark Granovetter (1973) sobre los lazos débiles. Para este autor los grupos más interrelacionados se caracterizan por establecer numerosos vínculos de menor intensidad y contacto físico o emocional a los que denomina débiles. Así, las tecnologías como Internet promueven conectividad a través de la creación de esta clase de vínculos. Por otra parte, relevamos la densidad de los hipervínculos presentes en los sitios seleccionados a partir de los programas informáticos Navicrawler y Gephi utilizados por el proyecto e-Diásporas Atlas de Dana Diminescu (2012). Siguiendo este protocolo, luego del relevamiento manual de los portales de las asociaciones de migrantes, los ingresamos en el programa Navicrawler con el objetivo examinar la estructura y los enlaces de cada uno de éstos y crear una base de datos que importamos al programa Gephi. Éste nos permitió graficar, visualizar e interpretar la estructura y distribución de las asociaciones de migrantes de países andinos en el ciberespacio.

\section{MIGRANTES ANDINOS EN LA WEB}

Si bien existen diversas periodizaciones, tradicionalmente se comprende que las migraciones de países andinos datan del periodo anterior a la Colonia (PELLEGRINO, 2010), pero se han proyectado hacia la Argentina fuertemente desde la década de 1990. Sus principales exponentes son los bolivianos, peruanos, colombianos y ecuatorianos. Ya señalamos que estos colectivos exponen dos tipos de migración, una reciente y joven colombianos y ecuatorianos- y otra tradicional y afincada -bolivianos y peruanos-.

Los bolivianos constituyen una de las colectividades migratorias sudamericanas más numerosas de la Argentina. Nuestro país es el primer destino elegido por éstos para emigrar, situación que se ve reflejada en su amplia distribución territorial y en su flujo constante. Su presencia es significativa en la ciudad de Buenos Aires, en el Gran Buenos Aires y en algunas ciudades del interior como Córdoba, San Salvador de Jujuy y Mendoza. En la actualidad se dedican a diversas actividades entre las que se destacan la industria de indumentaria y la horticultura (SASSONE, 2007).

La migración peruana data de 1950, pero se incrementó considerablemente en la década de 1990 motivada por la alta desocupación conse-

Rev. Cad.Comun, Santa Maria, v.20,n.1, art 1, p.1 de 24, jan/abr.2016 
cuencia de la implementación de un modelo caracterizado por políticas neoliberales en el Perú. Asimismo, fue un factor decisivo la violencia política que sufría aquel país resultado de los enfrentamientos entre las fuerzas estatales y Sendero Luminoso (ALTAMIRANO, 2010; LAPENDA, 2010). La colectividad peruana ha crecido sustancialmente en los últimos años y ha diversificado sus actividades, por ejemplo, la industria gastronómica (MELELLA, 2013). Su lugar de establecimiento resulta más concentrado que en el caso boliviano y su ciudad preferencial es Buenos Aires.

Los ecuatorianos y colombianos conforman una migración actual y con menor flujo que las anteriores, pero que muestra índices de crecimiento. Su perfil está compuesto, fundamentalmente, por estudiantes que viajan para perfeccionarse a las universidades argentinas, por consiguiente, la Ciudad de Buenos Aires, Córdoba y La Plata son sus distritos de radicación.

Hay que subrayar que los bolivianos y los peruanos en la Argentina poseen centenares de asociaciones, pero una cantidad inferior se extiende al ciberespacio. Los ecuatorianos y colombianos, al constituir una migración más reciente compuesta por gran cantidad de jóvenes estudiantes que suponemos nativos digitales (PISCITELLI, 2005), poseen una menor cantidad de asociaciones físicas pero su gran mayoría tiene presencia en la Web. Durante el año 2013, hemos relevado 48 sitios Web de asociaciones de la colectividad boliviana con dominio argentino, 55 de la peruana, 8 de la colombiana y 3 de la ecuatoriana. Las páginas Web de asociaciones pueden ser clasificadas como asociaciones civiles, culturales, comerciales, institucionales y medios de comunicación. Igualmente, estas asociaciones de migrantes pueden ser divididas en dos tipos a partir de su modo de acceso a la red. Por un lado, aquellas que existían previamente y luego desembarcaron en Internet y que comprendemos bajo el calificativo de "inmigrantes digitales". Por otra parte, se encuentran los grupos que tienen presencia en Internet y no poseen un espacio físico regular en el que desarrollen sus funciones. Éstos últimos fueron entendidos como "nativos digitales". Las asociaciones de migrantes andinos más arraigadas en la Argentina como los bolivianos y los peruanos se corresponden con el primer tipo y los ecuatorianos y colombianos, menos establecidos, pertenecen al segundo. A continuación expondremos un ejemplo de cada uno de ellos. 


\section{LA PRESENCIA DE ASOCIACIONES DE MIGRANTES ANDINOS EN LA WEB. CONECTIVIDAD Y CREACIÓN DE LAZOS COMUNITARIOS}

\section{EL CASO PERUANO}

El Consulado del Perú en la Argentina posee un registro de una treintena de asociaciones en todo el país. Sin embargo, como marcamos inicialmente, la presencia de asociaciones en la Web supera ampliamente esta cifra. Contrario a las asociaciones bolivianas en la Web que mantienen equidad con respeto a su tipo (culturales, civiles y medios de comunicación), los sitios Web que más predominan entre los peruanos son comerciales, en especial, de restaurantes (31 sitios sobre 55). Éstos utilizan Internet para promocionarse y acrecentar la clientela. Le siguen los sitios de medios de comunicación ( 11 de 55) y las asociaciones civiles (8 de 55).

Casa del Perú es una asociación civil sin fines de lucro cuya misión es organizar obras culturales, artísticas y de bienestar social (salud, vivienda y asesoramiento profesional) (Figura 1). Su objetivo es estimular la integración de los migrantes y residentes peruanos a la sociedad argentina. Si bien, ésta fue creada en la Ciudad Autónoma de Buenos Aires en 2009, sus integrantes y miembros fundadores son peruanos que han inmigrado a la Argentina en las décadas de 1950 y 1960. Esta situación los ubica en un lugar privilegiado para contribuir a que las nuevas generaciones de migrantes puedan participar de la sociedad receptora y de su colectividad de una forma más fructuosa y menos traumática. Estudiamos el sitio Web de esta asociación representativa de la colectividad peruana a partir de las tres dimensiones analíticas propuestas en la metodología:

I- Análisis textual: El diseño de la página principal condensa estrategias gráficas, ya que posee varias etiquetas de entrada que abordan los intereses y las temáticas que se consideran relevantes ('metodología', 'proyectos', 'voluntariado', entre otras). Su diagramación formal brinda al sitio un aire de solemnidad. Sin embargo, su logotipo -una tuerca- remite a la idea de construcción de una comunidad más sólida. Advertimos que el predominio del color rojo nos conecta metonímicamente al universo de la peruanidad a través de los tonos de su bandera nacional. Por último, las fotografías documentan las actividades llevadas a cabo por la asociación y no exhibe publicidades, condición que denota autonomía. 


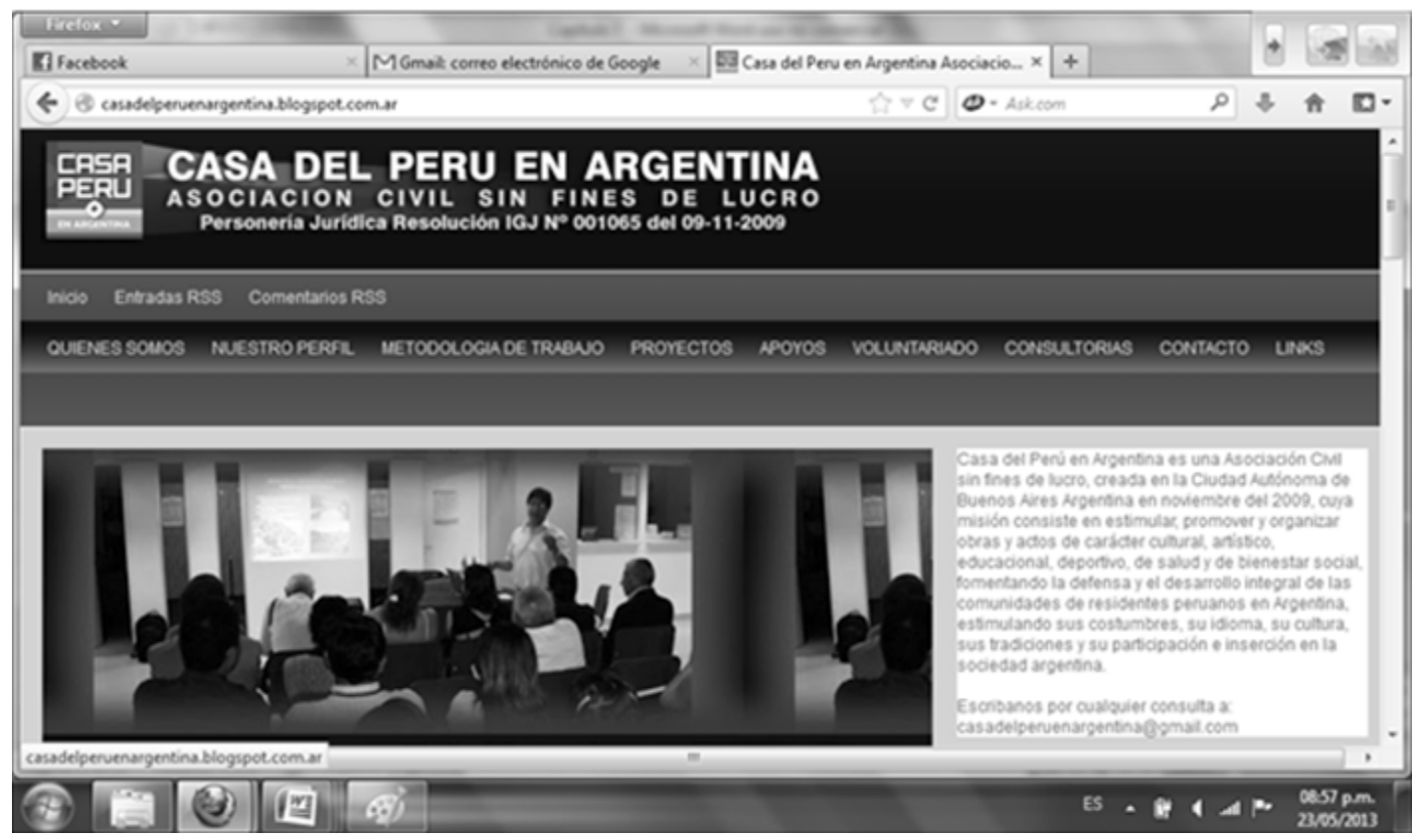

Figura 1 Sitio Web Casa del Perú

Fuente: casadelperuenargentina.blogspot.com

El tema que predomina es la solidaridad y se manifiesta en la concreción de proyectos culturales, habitacionales y sanitarios (ampliación de derechos civiles y sociales). Otras temáticas sobresalientes refieren a la unificación de la colectividad, a la circulación de la cultura peruana y a la transmisión de las experiencias de los residentes en la Argentina hacia las nuevas generaciones. En consecuencia, Casa del Perú se posiciona, por un lado, como un enunciador pedagógico que conduce al grupo hacia la unión en el seno de la sociedad receptora. Por otro lado, construye una relación basada en la complicidad entre enunciador y destinatario (potenciales lectores-usuarios) que comparten códigos y generan lazos -solidarios-.

II- Análisis estructural: Sobre la caracterización analítica desarrollada en el punto 2, advertimos que los contenidos informativos de este sitio se centran en las actividades propuestas por la asociación y exceptúan noticias generales tomadas de otros sitios (Figura 2). Los servicios al lector-usuario que consideran el uso de dispositivos y herramientas tecnológicas son escasos: archivo del blog y un buscador de noticias interno. Por su parte, las áreas para la participación para el lector-usuario también son exiguas al incluir exclusivamente "la carta de lectores". Por último, los recursos audiovisuales, multimedia e hipervínculos contemplan una cuenta de Facebook, enlaces y audio y video on streaming, término que

Rev. Cad.Comun, Santa Maria, v.20,n.1, art 1, p.1 de 24, jan/abr.2016 
hace referencia al hecho de escuchar música o ver vídeos sin necesidad de descargarlos.

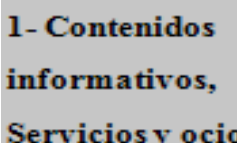

\begin{tabular}{|c|c|c|}
\hline & CASA DEL PERÚ & $\begin{array}{l}\text { ECUATORIANOS } \\
\text { EN ARGENTINA }\end{array}$ \\
\hline Últimas noticias & $\begin{array}{l}\text { Si, activida des de la } \\
\text { semana }\end{array}$ & No \\
\hline Pronóstico & No & No \\
\hline Transportes & No & $\mathrm{Si}$ \\
\hline Espectáculo & $\mathrm{Si}$, cultural & $\mathrm{Si}$, de la colectividad \\
\hline Direcciones útiles & $\mathrm{Si}$ & $\begin{array}{l}\text { Si, Emergencias, } \\
\text { Aeropuertos, } \\
\text { Hospitales, Cia, de } \\
\text { celulares, Embajada. }\end{array}$ \\
\hline Juegos & No & No \\
\hline Concursos & No & No \\
\hline Música & No & No \\
\hline $\begin{array}{l}\text { Información } \\
\text { institucional }\end{array}$ & $\begin{array}{l}\text { Si, Misión, } \\
\text { autoridades }\end{array}$ & $\mathrm{Si}$ \\
\hline
\end{tabular}

\begin{tabular}{l|l|l|l}
\hline \multirow{4}{*}{ 2-Servicios } & Personalización & No & No \\
\hline Hemeróteca & No & No \\
\hline Archivo & Si, del blog & Si, del blog \\
\hline Carpeta personal & No & No \\
\hline Barra de navegación & No & No \\
\hline Buscador de noticias & Si, intemo & Si, intemo \\
\hline Contenidos móviles & No & No \\
\hline \multirow{2}{*}{ Publicidad } & No & Si. Restaurantes \\
\hline Tienda virtual & No & ecuatorianos \\
\hline Clasificados & No & No \\
\hline Promociones & No & No
\end{tabular}




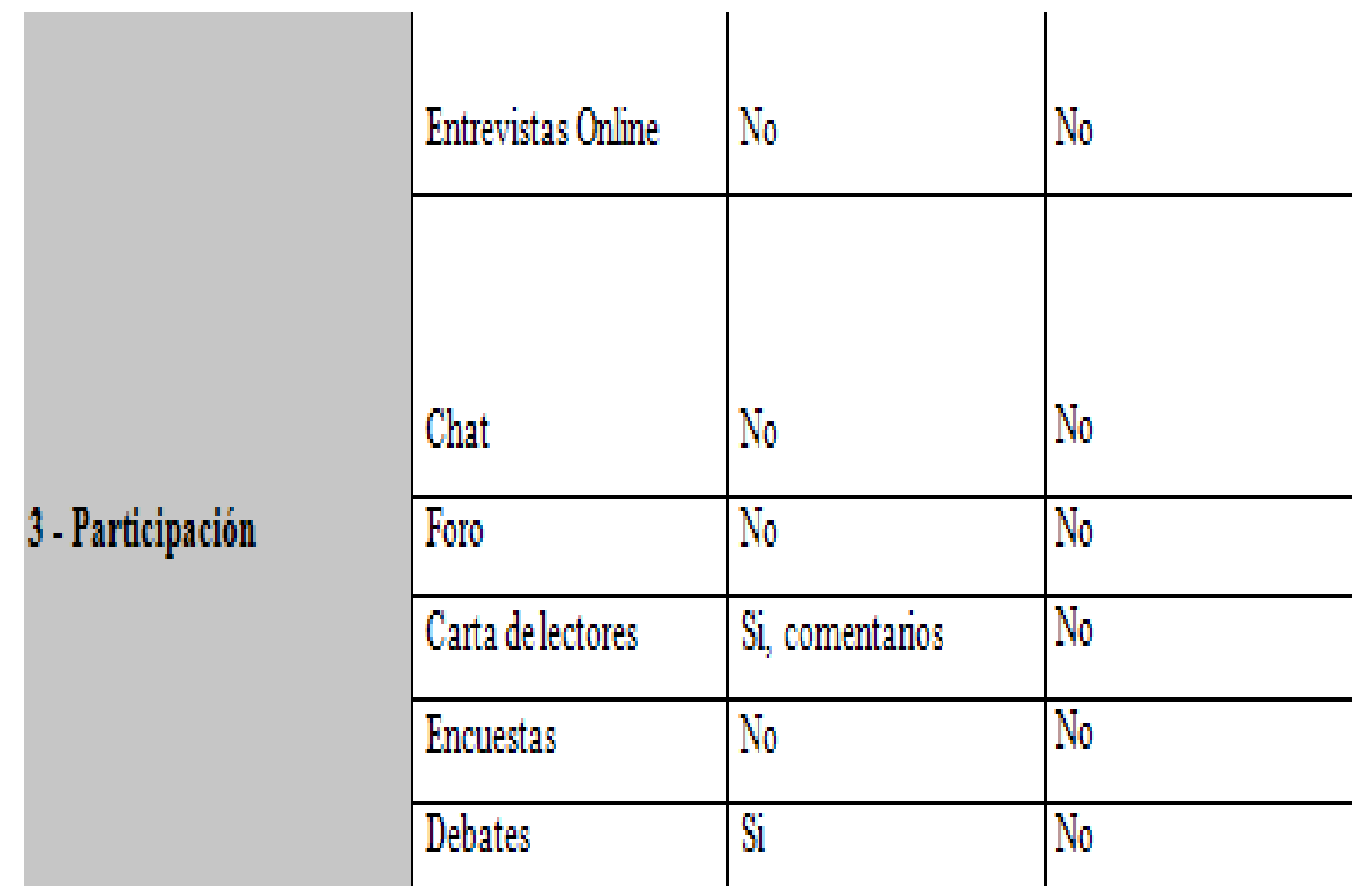

\begin{tabular}{|c|c|c|c|}
\hline & Enlaces & $\mathrm{Si}$ & $\begin{array}{l}\text { Si. Estudios en Arg., } \\
\text { Migración a Arg., } \\
\text { Ministerio de } \\
\text { Relaciones Exteriores, } \\
\text { Arg. Info gral. }\end{array}$ \\
\hline \multirow{6}{*}{4 - Recursos } & Web 2.0 & $\mathrm{Si}$, Facebook & $\begin{array}{l}\text { Si, Facebook, Pag. } \\
\text { Ecuatorianasy Red de } \\
\text { profesionales } \\
\text { ecuatorianos en Arg. }\end{array}$ \\
\hline & Gráficos & No & No \\
\hline & Audio On Demand & No & No \\
\hline & Audio On Streaming & $\mathrm{Si}$ & $\mathrm{Si}$ \\
\hline & Video On Demand & No & No \\
\hline & Video On Streaming & $\mathrm{Si}$ & $\mathrm{Si}$ \\
\hline
\end{tabular}

Figura 2 Estructura del sitio Casa del Perú y Ecuatorianos en Argentina 
III- Análisis de conectividad: Finalmente, se evidencia en el grafo de conectividad del ciber-espectro peruano en la Argentina (Figura 3) que sitios de asociaciones como Casa del Perú, Asociación de gastronomía peruana (AGAPERÚ), Mujeres Unidas, Migrantes y Refugiadas en Argentina (AMUMRA) y la página del Consulado Nacional del Perú son aquellos que generan más conectividad al fomentar hipervínculos. Estas asociaciones en la Web se convierten en las vehiculizadoras de redes al establecerse como puentes (lazos débiles según la terminología de Granovetter) que trascienden la comunicación cara a cara y evidencian aquello que Carlos Scolari (2008) define como hipermediaciones, es decir, "procesos de intercambio, producción y consumo simbólico que se desarrollan en un entorno caracterizado por una gran cantidad de sujetos, medios y lenguajes interconectados tecnológicamente de manera reticular entre sí" (SCOLA$\mathrm{RI}, 2008: 113)$. En el caso peruano, dada la presencia física de las entidades, las comunidades virtuales tienen el cometido de afianzar los lazos preexistentes.

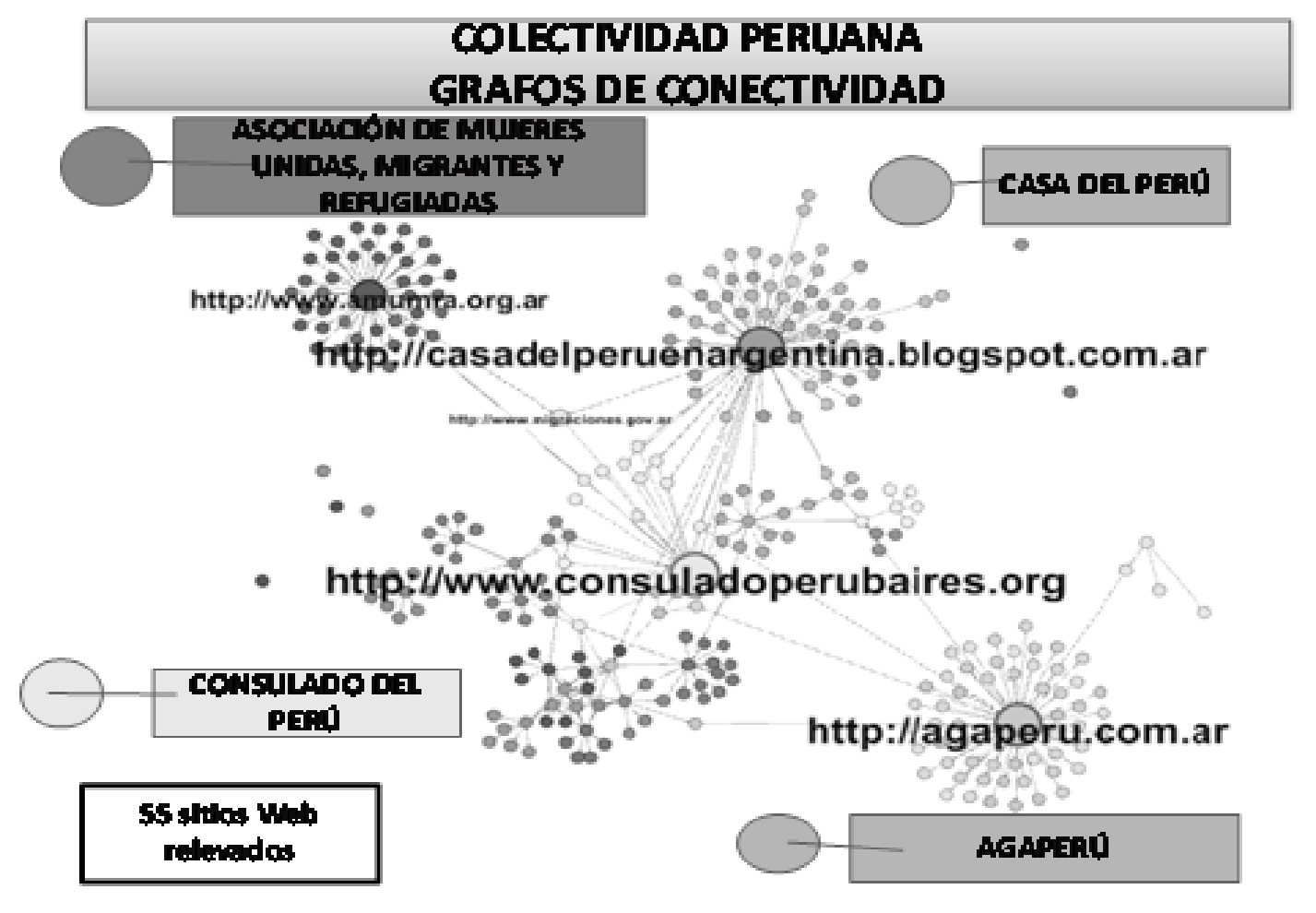

Figura 3 Grafos de conectividad Casa del Perú

Fuente: Elaboración de base de datos Cecilia Melella. Manejo de Gephi Daniel Hereñú

Rev. Cad.Comun, Santa Maria, v.20,n.1, art 1, p.1 de 24, jan/abr.2016 


\section{EL CASO ECUATORIANO}

Los ecuatorianos en la Argentina poseen pocas instituciones que excedan la Embajada y el Consulado del Ecuador. De las tres páginas recabadas se destaca Ecuatorianos en Argentina, un referente para este grupo. El portal fue creado por Fernanda Iglesias, una estudiante de Diseño y Publicidad de la Universidad de Palermo en el año 2009 (Figura 4). Ecuatorianos comenzó con un grupo de Facebook que se proponía brindar un servicio informativo a los connacionales que llegaban por primera vez a la Argentina.

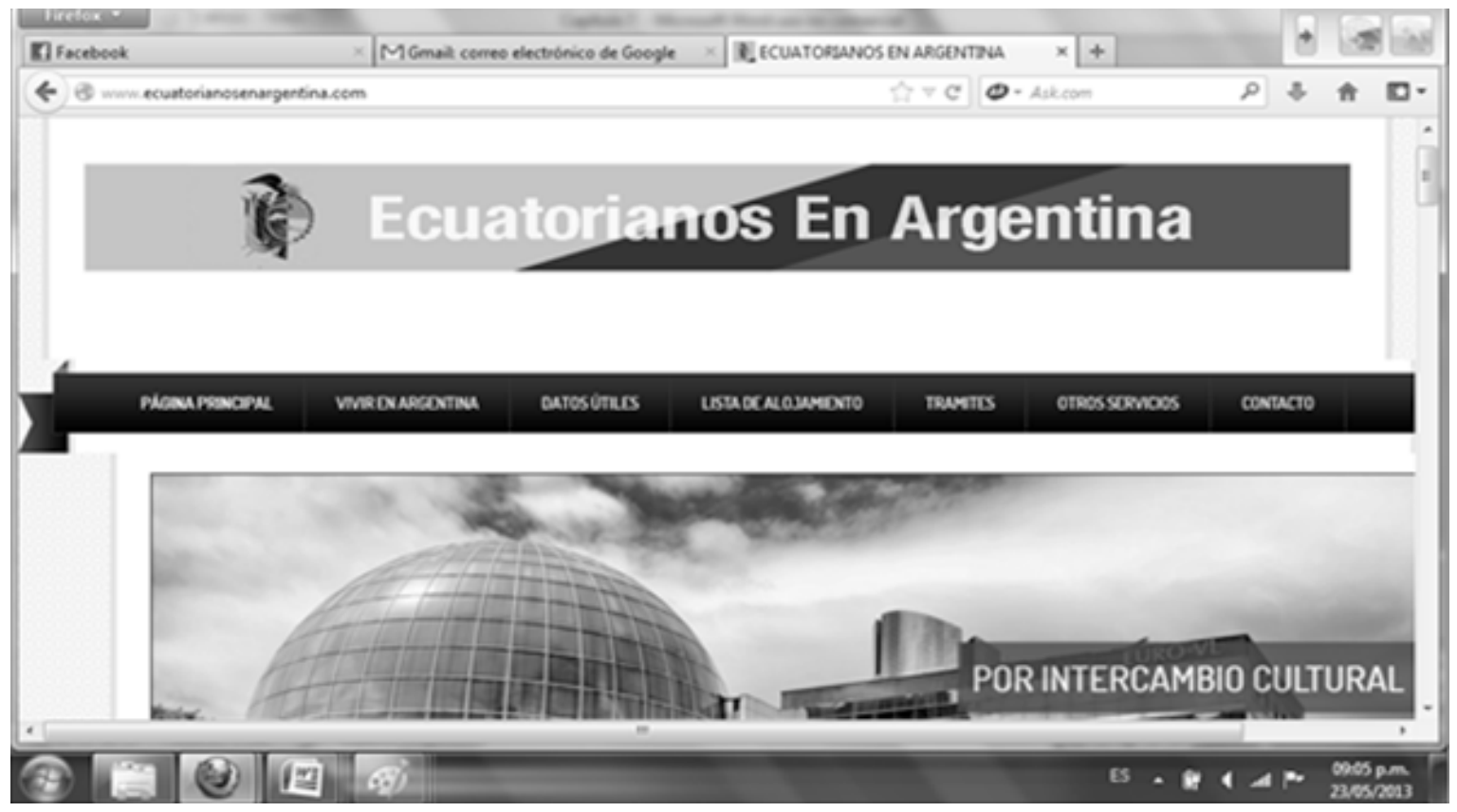

Figura 4 Ecuatorianos en Argentina

Fuente: www.ecuatorianosenargentina.com

Consecuencia de su creciente demanda, sus fundadores decidieron volcar la información a una página Web. Así, la creación de Ecuatorianos en Argentina es la materialización de las redes que tejen estos jóvenes que llegan para estudiar a nuestro país. Como en el caso peruano, la página también se establece como lazo que conecta a los pequeños grupos de ecuatorianos que residen en otras ciudades de la Argentina como La Plata y Córdoba vinculando los espacios on line y off line (MELELLA, 2014).

I. Análisis textual: Retomamos el análisis textual bajo una perspectiva semiótica basada en la caracterización de rasgos. El sitio Web se organiza

Rev. Cad.Comun, Santa Maria, v.20,n.1, art 1, p.1 de 24, jan/abr.2016 
a partir de estrategias gráficas que incluyen etiquetas que permiten diversas entradas y vehiculizan la búsqueda de información. Además, estas pestañas se corresponden con las temáticas abordadas por la asociación. Por ejemplo, se destacan entradas como 'Vivir en Argentina', 'Datos útiles', 'Lista de alojamientos', 'Trámites', 'Contacto', etcétera. Asimismo, hay presencia de colores de la bandera de Ecuador y fotografías de espacios emblemáticos de Buenos Aires como el Planetario y el barrio cosmopolita de Puerto Madero, que colocan en primer plano las dimensiones del aquí y del allá. La página incluye escasas publicidades que remiten directamente a la colectividad como los restaurantes étnicos Rincón Ecuatoriano y Ecuador 787. Por último, Ecuatorianos no cuenta con logotipo, sino que se centra en la inclusión de información cultural, deportiva y de servicios al inmigrante. El mismo sitio se definen como: “(... ) un espacio que concentra todo tipo de información que pueda aportar en tu estadía en Argentina. Toda la información ingresada son aportes desde la experiencia por Ecuatorianos para Ecuatorianos".

Los temas preponderantes versan sobre las políticas migratorias, la solidaridad, la circulación de expresiones culturales como tácticas de inserción política, cultural, civil en la sociedad de destino y la generación de lazos para el encuentro comunitario. Por último, Ecuatorianos en Argentina, construye, al igual que Casa del Perú, un enunciador cómplice y pedagógico que comunica sus propias experiencias y ofrece asistencia a los recién llegados.

II- Análisis estructural: Ecuatorianos en Argentina propone diversidad de contendidos informativos dispuestos para la orientación del inmigrante (Figura 2). En consecuencia, no proporciona variedad contenidos para el ocio. También, las herramientas de servicios se apuntan a ofrecer información y son escasos los elementos que contemplen un área de participación on line. Los recursos audiovisuales, multimedia e hipervínculos incluyen links, Web 2.0 y audio video on streaming.

III- Análisis de conectividad: Las asociaciones ecuatorianas son "nativas digitales" pero se proyectan sobre el espacio off line con actividades como campeonatos de fútbol o los eventos de Net Working, programas públicos que establecen contactos entre profesionales con objetivo de organizar una red. Asimismo, tiene una función informativa para la colectividad. Son organizados por Andrea (Red de Profesionales Ecuatorianos en la Argentina). Así, el grafo de conectividad (Figura 5) demuestra que, tal

Rev. Cad.Comun, Santa Maria, v.20,n.1, art 1, p.1 de 24, jan/abr.2016 
como en el ejemplo peruano, los sitios Web Ecuatorianos en Argentina y Estudiar en Argentina son los que crean lazos de conectividad al establecer más hipervínculos.

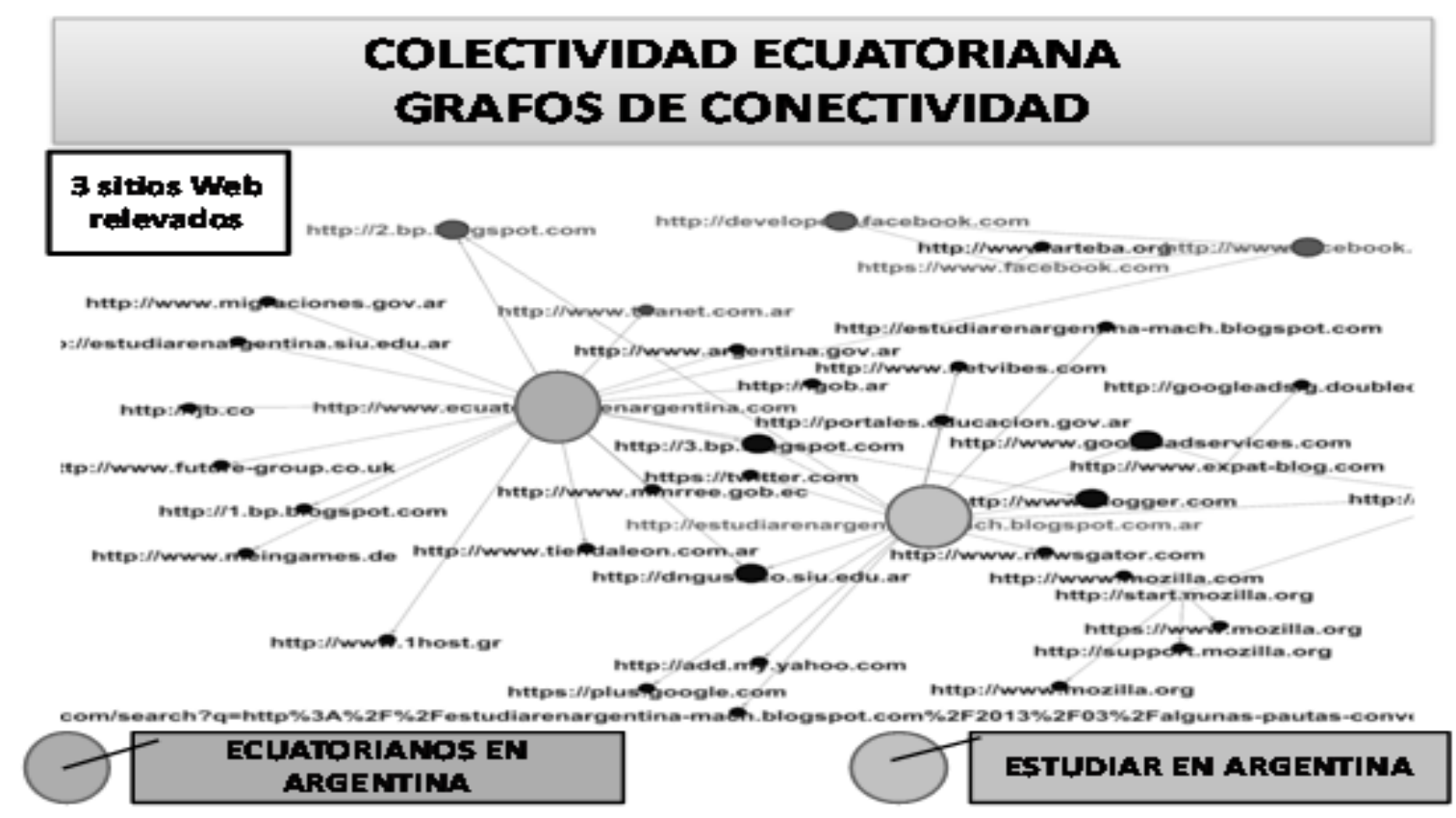

Figura 5 Grafo de conectividad Ecuatorianos en Argentina

Fuente: Elaboración de base de datos Cecilia Melella. Manejo de Gephi Daniel Hereñú

\section{REFLEXIONES FINALES}

Los sitios Web analizados evidencian el cambio del concepto de comunidad que pasa del lugar físico a la red. A partir de las tres dimensiones analíticas concluimos que, desde el estudio semiótico, en estos sitios prevalece un vínculo simbólico con el origen (referencias a los Estados nacionales a través de sus banderas). Sin embargo, los temas se vinculan al aquí y se proponen brindar información y servicios al recién llegado, fomentar la solidaridad y la creación de lazos comunitarios. Así, ambos portales establecen una relación con el destinatario basada en un contrato pedagógico y cómplice.

Asimismo, observamos que el objetivo de brindar información y servicio se sustenta en la estructura tecnológica al privilegiar los contenidos informativos por sobre los de ocio. Los servicios también se orientan a ese objetivo (buscadores internos y archivos del blog). Tampoco se ahonda en la participación, sino que se mantiene la lógica del punto a masa característica de los medios de comunicación tradicionales como la prensa gráfica

Rev. Cad.Comun, Santa Maria, v.20,n.1, art 1, p.1 de 24, jan/abr.2016 
o la televisión.

No obstante, hemos demostrado a través del uso de los programas Navicrawler y Gephi que las comunidades que forman nodos son las que se encuentran más conectadas con otros sitios, es decir, poseen más hipervínculos. Tal es el caso de Casa del Perú y Ecuatorianos en la Argentina. Estas asociaciones gozan de un mayor poder de arraigo y se convierten en las vehiculizadoras de redes. En este sentido, las comunidades "nativas digitales" crean lazos y las "inmigrantes digitales" afianzan los existentes. La presencia de asociaciones en la Web genera hipermediaciones e instaura una nueva tecnosocibilidad en el migrar (más evidente en el caso ecuatoriano). Se establecen como espacios de articulación inter e intra-comunitarios y contribuyen a la creación y el funcionamiento de redes sociales de migrantes. 
CADERNOS DE COMUNICAÇÃO

UNIVERSIDADE FEDERAL DE SANTA MARIA

\section{REFERENCIAS}

ALBORNOZ, Luis. Periodismo Digital. Los Grandes Diarios en la Red. La Crujía. Buenos Aires, 2007.

ALTAMIRANO, Teófilo (2010). Migración internacional, transferencias y remesas. El caso peruano. Oteiza E. (comp.) Patrones migratorios en América Latina. Buenos Aires: EUDEBA, 2010.

BARRANTES, R, JORDÁN, V. Y ROJAS, F. La evolución del paradigma digital en América Latina. En Jordán, Galperín y Perez (comps.). Banda ancha en América Latina: más allá de la conectividad. Santiago de Chile: CEPAL, 2013.

BARTHES, Roland. Lo obvio y lo obtuso. Paidós. Buenos Aires, 1986.

CASTELLS, Manuel. La Era de la Información. Vol. I: La Sociedad Red. Alianza. Madrid, 1995.

DIMINESCU, Dana (2011). El migrante conectado. Por un manifiesto epistemológico. Hernández, V., Mera, C. y Oteiza, E. (comp.) Circulación de saberes y movilidades internacionales: Perspectiva latinoamericana. Buenos Aires: Biblos, 2011.

DIMINESCU, Dana. E-Disporas Atlas. Exploration and Cartography of Diasporas in Digital Networks. Recuperado el 15 de marzo de 2014, de http://www.inter-disciplinary.net/ at-the-interface/wp-content/uploads/2012/06/diminescudpaper.pdf. 2012.

ESTALELLA, Adolfo y ARDÉVOL, Elisenda. Internet: instrumento de investigación y campo de estudio para la antropología visual. Revista chilena de Antropología Visual, 15, pp. 1-21, 2010.

FINQUELIEVICH, Susana. Del café de barrio a las redes electrónicas. Las comunidades virtuales como actores sociales en las ciudades. Recuperado el 01 de junio de 2013, de http://www.revistakairos.org/ko4-do2.htm, 1999.

GÓMEZ ESCALONILLA, Gloria y María Victoria CAMPOS ZABALA. Espacios de solidaridad en Internet: webs para inmigrantes. Recuperado el 10 de mayo de 2013, de http:// migraciones.ugr.es/omecia/pages/seminarios/textos/Inmigraci\%C3\%B3n\%20e\%2olnternet.pdf, 2009.

Rev. Cad.Comun, Santa Maria, v.20,n.1, art 1, p.1 de 24, jan/abr.2016 
GONZÁLVEZ TORRALBO, Herminia. (2012). Comunicación y contacto transnacional: El uso de las nuevas tecnologías de información y comunicación en la constitución de redes migratorias. Revilla Blanco, M. y Gómez Johnson, C. (eds.) Caminos de ida y vuelta: redes, migración y desarrollo. Madrid: Catarata, 2012.

GRANOVETTER, Mark. The strength of weak ties. American Journal of Sociology, vol. 78. 6, pp. $1360-1380,1973$.

HINE, Christine. Etnografía virtual. UOC. Barcelona, 2004.

KISSAU, Katrhrin y HUNGER, Uwe (2010). The internet as a means of studying transnationalism and diaspora. Bauböck y Faist (eds.) Diaspora and Transnationalism: Concepts, Theories and Methods. Amsterdam: Amsterdam University Press, 2010.

LAPENDA, Marina. Migrantes peruanos en la ciudad de Buenos Aires: Configuración de espacios de vida y espacios vividos. Tesis de licenciatura no publicada, Universidad Nacional del Centro de la Provincia de Buenos Aires, Facultad de Humanidades, Tandil, Argentina, 2010.

MARTÍN-BARBERO, Jesús. De los medios a las mediaciones. Comunicación, cultura y hegemonía. Gustavo Gili Editores. Barcelona, 1991.

MELELLA, Cecilia. Migración y tecnologías de la información y la comunicación (TIC). De la prensa gráfica a las redes virtuales como espacios de construcción identitaria de los migrantes de países andinos en la Argentina. Tesis de doctorado no publicada, Universidad de Buenos Aires, Facultad de Ciencias Sociales, Buenos Aires, Argentina, 2013.

MELELLA, Cecilia. Migraciones emergentes hacia la Argentina. Colombianos y ecuatorianos. Breve panorama y estrategias de inserción cultural. Si Somos Americanos. Revista de Estudios Transfronterizos, Volumen XIV, 2, pp. 15-46, 2014.

PEÑARANDA CÓlERA, María del Carmen (2008) ¿Tecnologías que acercan distancias? Sobre los 'claroscuros' del estudios de la(s) tecnología(s) en los procesos migratorios transnacionales. Santamaría, E. (Ed.), Retos epistemológicos de las migraciones transnacionales Barcelona: Antrophos, 2008.

PELLEGRINO, Adela (2010). Tendencias de la migración internacional en América latina y El Caribe en la segunda mitad del siglo XX. Oteiza E. (comp.), Patrones migratorios 
CADERNOS DE COMUNICAÇÃO

UNIVERSIDADE FEDERAL DE SANTA MARIA

en América Latina. Buenos Aires: EUDEBA, 2010.

PISCITELLI, Alejandro. Meta-cultura, El eclipse de los medios masivos en la era de Internet. La Crujía. Buenos Aires, 2002.

PISCITELLI, Alejandro. Internet. Imprenta del siglo XXI. Gránica. Barcelona, 2005.

PORTES, Alejandro. Convergencias teóricas y evidencias empíricas en el estudio del transnacionalismo de los inmigrantes. Migración y desarrollo, 4, pp. 2-19, 2005.

QUINTAR, Aída (2007). Redes sociales y comunidades virtuales. Quintar, A; Calello, Ty Aprea, G. (comps.) Los usos de las TICs. Una mirada multidimensional. Buenos Aires: Prometeo, 2007.

SASSONE, Susana. Migración, territorio e identidad cultural: construcción de lugares bolivianos en la ciudad de Buenos Aires. Revista Población de Buenos Aires, 4, pp. 9-28, 2007.

SCOLARI, Carlos. Hipermediaciones. Elementos para una teoría de la comunicación digital interactiva. Gedisa. Barcelona, 2008.

STEIMBERG, Oscar. Semiótica de los medios masivos. Atuel. Buenos Aires, 1993.

VERÓN, Eliseo. El análisis del "Contrato de Lectura”, un nuevo método para los estudios de posicionamiento de los soportes de los media. Les Medias: Experiences, recherches actuelles, aplication, 1985

VERÓN, Eliseo. La semiosis social. Fragmentos de una teoría de la discursividad. Gedisa. Barcelona, 1993.

WINOCUR, Rosalía. Apropiación de Internet y la computadora en sectores populares urbanos. Versión, 19, pp. 191-216, 2007.

WINOCUR, Rosalía. Robinson ya tiene celular. Siglo XXI. México, 2010.

Rev. Cad.Comun, Santa Maria, v.20,n.1, art 1, p.1 de 24, jan/abr.2016 


\section{Cecilia Melella}

Dra. en Ciencias Sociales y Magister en Comunicación y Cultura por la Universidad de Buenos Aires (UBA), Licenciada y Profesora en Educación Superior en Ciencias de la Comunicación (UBA). Docente de Ciclo Básico Común (UBA). Becaria posdoctoral del Consejo Nacional de Investigaciones Científicas y Técnicas (CONICET). Miembro del Proyecto de Investigación Plurianual “Migración internacional en ciudades de la argentina: lugares, territorios e identidades en la era de la globalización" IMHICIHU-CONICET e investigador junior en el proyecto GOVDIV Multilevel governance of cultural diversity in a comparative perspective: EU-Latin America. Sus áreas de investigación son identidades-alteridades, migraciones, medios y tecnologías de la información y de la comunicación (TIC).

RECEBIDO EM: 08/02/2016

APROCADO PARA PUBLICAÇÃO: 18/03/2016 